\title{
IMPORTANCE OF THE MASCC SCORE IN THE APPROACH OF FEBRILE NEUTROPENIA
}

André Ferreira ${ }^{1}$, Mafalda Miranda Baleiras ${ }^{1}$, Maria Teresa Neves ${ }^{1}$, Mariana Malheiro ${ }^{1}$, Alice Sousa ${ }^{1}$, Ana Martins $^{1}$

${ }^{1}$ Hospital de São Francisco Xavier - Centro Hospitalar de Lisboa Ocidental, Portugal

\section{INTRODUCTION}

Febrile neutropenia (FN) is one of the most frequent and serious complications of cancer chemotherapy, with a profound impact on the evolution of patients with cancer, leading to treatment delay and dose modifications. ${ }^{1,2}$

Patients with FN should be assessed for the risk of complications using a validated predictive tool, such as the Multinational Association of Supportive Care in Cancer (MASCC) score. ${ }^{1} \mathrm{~A}$ cumulative MASCC score of $\geq 21$ points is strongly linked to an uneventful clinical course and is associated with low rates of bacteremia and medical complications. ${ }^{3}$

The objective of this study is to evaluate the applicability of the MASCC score in patients diagnosed with FN at the Emergency Department (ED) and the adequacy of the antibiotic and granulocyte colony-stimulating factors (G-CSF) instituted.

\section{METHODS}

Observational, retrospective and unicentric study of patients with solid malignant neoplasms diagnosed with FN at a Portuguese ED, during four years, between January 2014 and December 2017.

\section{RESULTS}

Of the 118 patients admitted to the ED with the diagnosis of neutropenia, 66 were excluded: 56 presented with haematological neoplasms, 3 presented with autoimmune disease and 7 presented with neutropenia without fever. Of the 52 patients included in the study, corresponding to those with solid malignant neoplasms diagnosed with FN at the ED, 57.7\% were females, with a median age of 66 years (30-84).

Figure 1 shows the localization of the primary tumor, being that $44.2 \%$ of patients presented with metastatic disease.

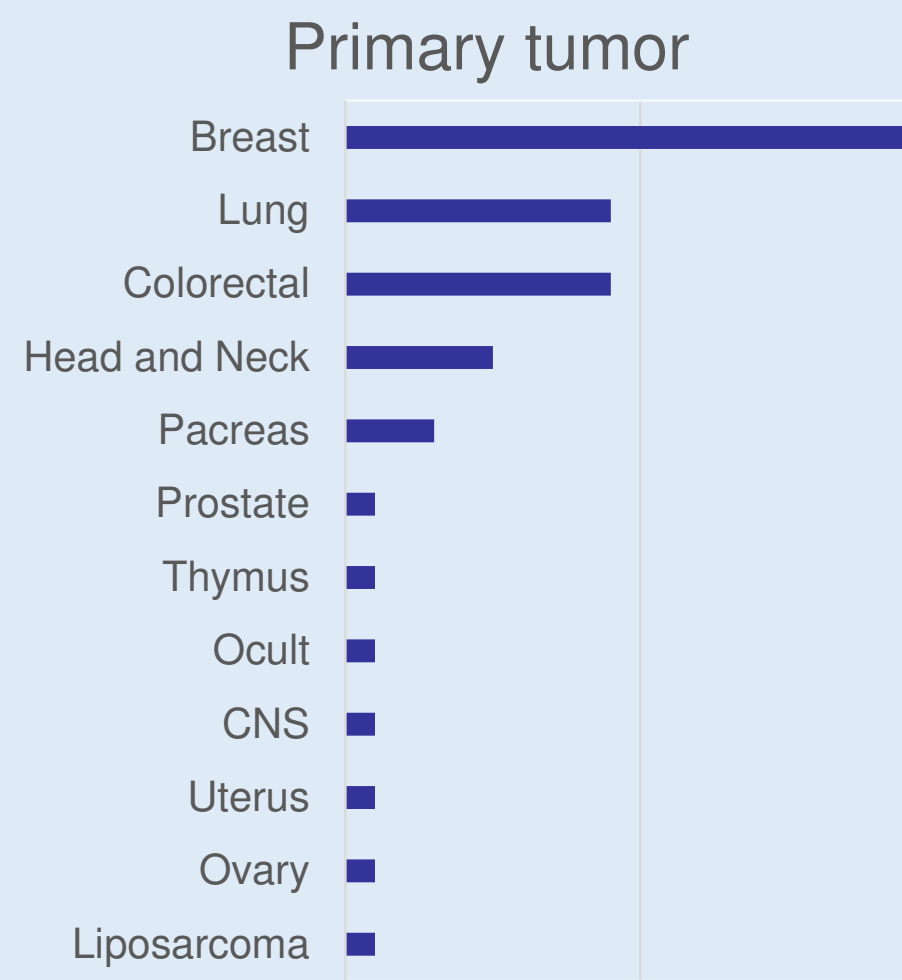

Figure 1.

The most commonly used chemotherapy regimens are shown in figure 2, with an average time between the last cycle and the ED admission of 10.4 days.

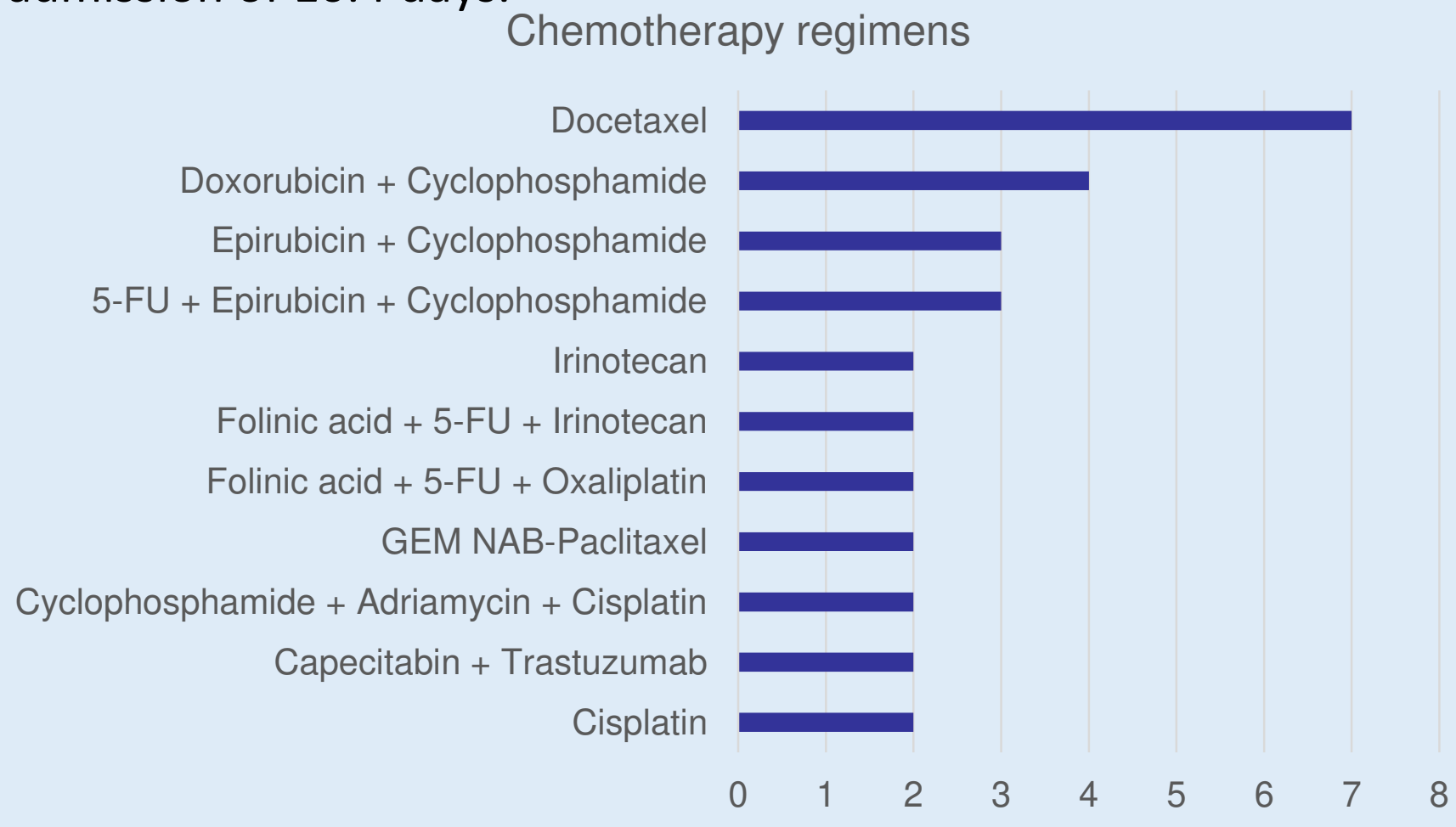

Figure 2 .
The MASCC score was applied in 9 patients (17.3\%). As shown in figure 3, 7 patients presented with low risk of complications, being that 5 of them were treated as inpatient with intravenous antibiotic therapy.

9 patients with MASCC score applied
7 patients with low

risk of complications (MASCC score $\geq 21$ )
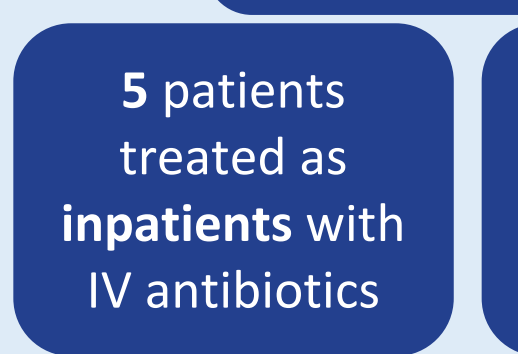

2 patients treated as outpatients with oral antibiotics
2 patients with high risk of complications (MASCC score <21)

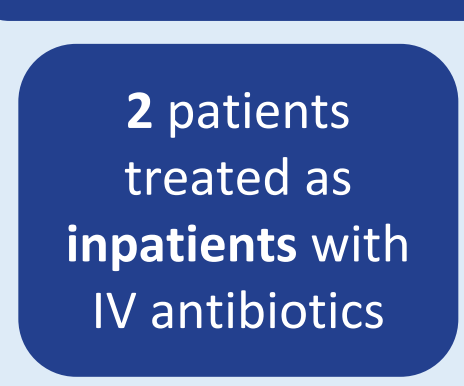

Figure 3.
The MASCC score of the remaining 43 patients was applied, $a$ posteriori, concluding that 22 patients presented at low risk of complications, in which 1 patient was treated as outpatient with oral antibiotic therapy (figure 4).

\section{3 patients without MASCC score applied}

22 patients with low risk of complications (MASCC score $\geq 21$ )
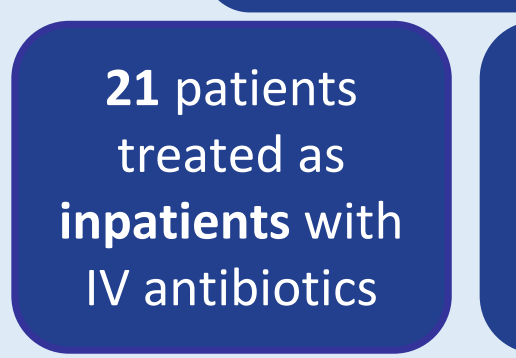

21 patients with high risk of complications (MASCC score <21)

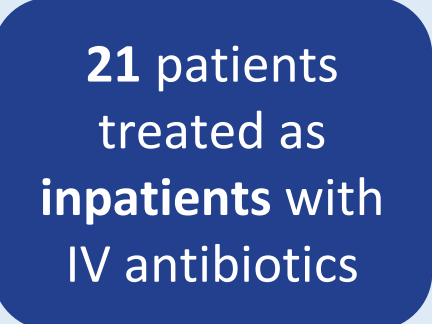

Figure 4.

All hospitalized patients received intravenous antibiotic therapy. The regimen most widely used was piperacillin/tazobactam plus an aminoglycoside (76.6\%). Blood cultures were positive in $17 \%$ of patients and the most common agent was a multisensitive Escherichia coli.

$85.7 \%$ of hospitalized patients were treated with G-CSF, with half presenting at low risk of complications.

\section{CONCLUSIONS}

This study aims to demonstrate that the MASCC score is rarely applied at the ED, in which practically all patients with FN are hospitalized for double broad-spectrum intravenous antibiotic therapy and for G-CSF treatment, including the majority of low-risk patients, despite the leading oncology societies recommended that patients with $\mathrm{FN}$ at a low risk of complications can often be treated with oral antibiotics and possibly as outpatients and that G-CSF should not be routinely used as adjuvant treatment with antibiotic therapy in patients with FN.

${ }^{1}$ Klastersky J, de Naurois J, Rolston K et al. Management of febrile neutropaenia: ESMO Clinical Practice Guidelines. Annals of Oncology. 2016 ; 27 (Supplement 5): v111-v118

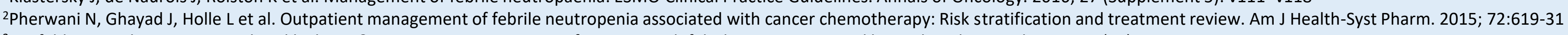
${ }^{3}$ Freifeld AG, Sepkowitz KA: No place like home? Outpatient management of patients with febrile neutropenia and low risk. J Clin Oncol. 2011; 29(30):3952-4 